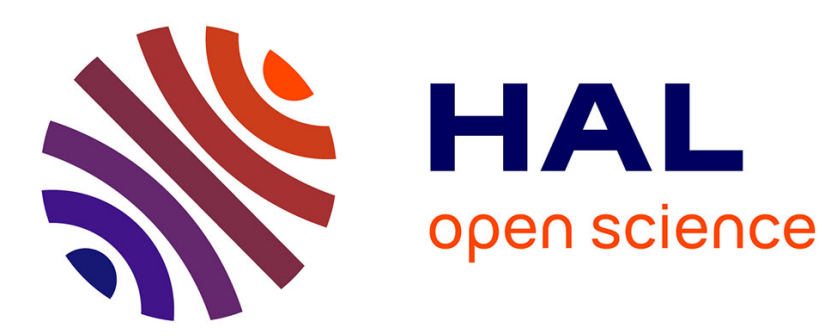

\title{
Contrôles et proximités au sein de la supply chain aéronautique
}

Damien Talbot

\section{To cite this version:}

Damien Talbot. Contrôles et proximités au sein de la supply chain aéronautique. Logistique \& Management, 2015, 19 (1), pp.3-14. 10.1080/12507970.2011.11516977 . hal-02351969

\section{HAL Id: hal-02351969 \\ https://hal.science/hal-02351969}

Submitted on 6 Nov 2019

HAL is a multi-disciplinary open access archive for the deposit and dissemination of scientific research documents, whether they are published or not. The documents may come from teaching and research institutions in France or abroad, or from public or private research centers.
L'archive ouverte pluridisciplinaire HAL, est destinée au dépôt et à la diffusion de documents scientifiques de niveau recherche, publiés ou non, émanant des établissements d'enseignement et de recherche français ou étrangers, des laboratoires publics ou privés. 


\title{
Contrôles et proximités au sein de la supply chain aéronautique
}

\author{
Damien Talbot \\ GREThA (UMR CNRS 5113) \\ Université de Bordeaux, Avenue Léon Duguit, 33608 Pessac Cedex \\ damien.talbot@u-bordeaux4.fr
}

Article paru dans Logistique \& Management

2011, vol. 19, n²1, décembre 2011, pp. 3-17.

\begin{abstract}
Résumé
Si les modes de coordination utilisés par les firmes au sein de la supply chain doivent permettre de transmettre des connaissances et de combiner des compétences, ils conduisent aussi à révéler les capacités techniques, financières et organisationnelles des parties prenantes, à vérifier si les engagements pris sont tenus, à réduire le risque de comportements opportunistes. Ainsi, les outils de coordinations inter-firmes visent, au-delà du seul partage de connaissances, au contrôle du partenaire. Si ces résultats sont connus, plus rarement a été exploré le rôle que pouvait jouer la proximité sur le contrôle de la relation donneurspreneurs d'ordres. L'objectif de cet article est de répondre aux questions suivantes : la proximité dans ses diverses dimensions favorise-t-elle le contrôle? Si oui par quels mécanismes? Nous montrons que le contrôle, qu'il soit formel ou informel, s'accompagne dans le cas que nous avons observé d'une proximité organisationnelle entre le contrôleur et le contrôlé, tandis que la proximité géographique peut venir renforcer ce même contrôle. Ces observations ont été réalisées au sein de la supply chain aéronautique. En particulier sont observés les relations de contrôle entre architectes-intégrateurs et firmes-pivots.
\end{abstract}

Mots clés : proximité organisationnelle, proximité géographique, contrôle, firme-pivot, architecte-intégrateur, supply chain aéronautique. 


\section{INTRODUCTION}

Les modes de coordination mobilisés par les firmes appartenant à une même supply chain peuvent être analysés dans une perspective classique mettant en lumière des comportements coopératifs de partage de connaissances. Les coordinations inter-firmes visent alors à acquérir, conserver, combiner et transmettre des connaissances, des représentations, des savoir-faire, des expériences, des process, des compétences, etc. Simultanément, la mise en place des mêmes modes de coordination peut résulter de la volonté pour un acteur de contrôler le partenaire avec lequel il compte interagir. Les coordinations inter-firmes ont alors aussi pour but de révéler les capacités techniques, financières et organisationnelles des parties prenantes, de vérifier si les engagements pris sont tenus, de réduire le risque de comportements opportunistes et au final de générer la confiance nécessaire à la création et au maintien d'une relation productive. Dit autrement, les outils de coordinations inter-firmes au sein de la supply chain visent tout autant au transfert de connaissances qu'au contrôle du partenaire.

Si en Sciences de Gestion ces résultats sont connus (Ouchi, 1979 ; Van der Meer-Kooistra et Vosselman, 2001 ; Das et Teng, 2001 ; Dekker, 2004), plus rarement a été exploré le rôle que pouvait jouer la proximité sur le contrôle d'une relation de type donneurs-preneurs d'ordres. Dans leur dimension non géographique, l'étude des relations de proximités met à jour la variété des modalités de coordinations en insistant sur la nécessité du partage de représentations, de valeurs, de règles mais aussi sur le besoin de régulation des conflits, l'impact des rapports de pouvoir, etc. Au-delà, cette approche permet d'intégrer à l'analyse les effets de la proximité géographique sur les relations inter-firmes, afin que de la dimension géographique ne reste pas «un impensé de la gestion» (Lauriol et al, 2008, p. 92). L'abondante littérature sur les clusters a d'ores et déjà montré que la proximité géographique pouvait, lorsqu'elle est souhaitée, favoriser le transfert et le partage de connaissance. Elle peut aussi favoriser le contrôle du partenaire.

L'objectif de cet article est alors proposer des pistes de recherche pour répondre aux questions suivantes : la proximité dans ses diverses dimensions favorise-t-elle le contrôle ? Si oui par quels mécanismes? Pour répondre à ces questions, nous procédons en deux temps : nous étudions dans une première partie les relations de proximités existantes au sein de la supply chain aéronautique, puis dans une seconde partie nous introduisons la question du contrôle. Le choix de l'aéronautique s'explique par le poids du contrôle exercé par les donneurs 
d'ordres sur les preneurs d'ordres dans ce secteur, les premiers cherchant à contrôler les seconds, tandis que les seconds visent à s'émanciper des premiers. A cette forte asymétrie entre acteurs s'ajoute la question de l'innovation dans une industrie de hautes technologies, innovation souvent réalisée en commun tant les coûts de R\&D sont élevés et le champ des compétences à mobiliser étendu. Cette innovation collective, sujette à l'opportunisme, appelle un contrôle du partenaire. En ce sens, le secteur aéronautique constitue un terrain d'observation pertinent pour nos questionnements. Sont particulièrement observés, d'une part, les architectes-intégrateurs et, d'autre part, certains équipementiers de rang 1 qui présentent la particularité d'adopter la forme organisationnelle dite de « firme-pivot».

\section{LES PROXIMITES DANS LA SUPPLY CHAIN AERONAUTIQUE}

L'approche par la proximité part du principe que la localisation dans l'espace géographique et le positionnement dans un réseau d'interactions conditionnent les activités économiques des acteurs, dans le sens où les caractéristiques de l'insertion sociale et géographique ont vocation «à conditionner la probabilité d'apparition d'une relation ou celle du renforcement ou de l'affaiblissement d'une relation existante» (Pecqueur et Zimmermann, 2004, p. 20). L'approche vise alors à déterminer quelles sont les conditions à l'apparition et/ou au renforcement d'une relation. Selon cette démarche, l'acteur est présent à la fois «ici et ailleurs » (Rallet, 1999). Ici car il est localisé dans un espace géographique au sein duquel il entretient des relations de voisinage, ailleurs car l'acteur est évidemment en relation à distance avec d'autres agents. De fait, on peut être «proche» de quelqu'un tout en étant éloigné géographiquement: la proximité présente alors, au côté de la dimension géographique, une dimension non géographique permettant de poser en même temps la question de la localisation et de l'organisation. Par conséquent, la proximité sera comprise comme un fait institutionnel (Talbot, 2010) présentant deux dimensions, organisationnelle et géographique.

\subsection{La proximité organisationnelle...}

La proximité organisationnelle se déploie au sein des organisations comme les firmes, des réseaux d'alliances stratégiques, etc. Elle se construit sur une faible distance sociale, comme la proximité géographique qui se construit sur une faible distance métrique. Par distance sociale il est fait ici référence aux attributs des acteurs en termes de ressources cognitives et 
matérielles, leur âge, leur santé, leur sexe, etc., bref l'ensemble des critères objectifs qui permettent de classer les individus en groupes plus ou moins homogènes. La distance sociale concerne aussi des organisations, pensons aux firmes partageant les mêmes marchés, les mêmes technologies, les mêmes produits, les mêmes fournisseurs, les mêmes financeurs, etc.

Une faible distance sociale ne suffit pas à créer une proximité organisationnelle : de la même façon que deux voisins de pallier peuvent s'ignorer, deux entreprises appartenant à un même secteur peuvent n'entretenir aucune relation autre que de simples rapports indirects de concurrence. En ce sens, la proximité n'est pas le simple inverse de la distance et ne se réduit pas à une simple similitude de caractères. On est proche d'autrui lorsque, certes lorsqu'on lui ressemble, mais aussi lorsqu'on partage diverses ressources avec lui. On parlera de proximité organisationnelle entre des acteurs lorsqu'ils partagent, au-delà d'une similitude dans leurs structures juridiques et en dotations de facteurs, un langage, des valeurs morales, des normes sociales, des règles, des routines, des représentations, etc. mais aussi un projet collectif significatif d'une réflexion stratégique commune. Ces ressources cognitives vont faire l'objet d'un apprentissage de la part des membres de l'organisation ou du réseau. Il existe alors un processus d'accumulation de telles ressources en vue de réaliser des tâches de manière plus efficace et qui correspond à la construction d'une mémoire organisationnelle. Ces ressources cognitives, lorsqu'elles sont partagées, permette d'acquérir, de conserver et de transmettre des connaissances, des savoir-faire, des expériences, bref d'activer une relation : on passe alors d'une ressemblance à une connexion positive.

Mais le partage de ressources cognitives n'est pas la seule condition à l'établissement d'une relation. Il faut en sus donner une cohérence à des intérêts contradictoires, réguler des conflits toujours latents, hiérarchiser les problèmes, prendre et imposer des arbitrages concernant par exemple le partage des gains espérés de l'interaction ou encore élaborer des compromis. Dit autrement, il faut au côté des ressources cognitives, partager des outils de coordination visant à la régulation pour qu'une relation s'instaure. Ce travail de mise en cohérence permettra à des acteurs aux positions sociales et aux intérêts différents de s'intégrer dans la structure de pouvoir qui accompagnera la relation, en prenant une place dans la hiérarchie et en assumant le rôle afférant. Le partage souhaité ou imposé d'outils de régulation est la seconde condition à l'établissement d'une interaction. On trouve ainsi dans la supply chain aéronautique un exemple de déploiement d'une telle proximité organisationnelle. 


\section{2 ... dans la supply chain aéronautique ${ }^{1}$}

Depuis les années quatre-vingts, on assiste à la fois à une complexification croissante et à une extension du champ des savoirs mobilisés pour la conception et la construction d'un avion. Un avionneur ne peut dès lors maîtriser l'ensemble des systèmes. Du fait de ces évolutions, les principaux avionneurs (Bombardier, Embraer, Boeing et Airbus) opèrent un recentrage de leurs activités sur l'amont (conception, R\&D) et sur l'aval de la chaîne de valeur (assemblage, commercialisation et services associés en formation et assistance technique). Ce recentrage stratégique est rendu possible par un approfondissement de la modularisation du processus productif. La décomposition de l'avion en sous-ensembles techniquement homogènes (modules) confère ainsi à l'avionneur des fonctions nouvelles d'architecte (amont) et d'intégrateur (aval) des activités (Frenken, 2000 ; Brusoni et Prencipe, 2001 ; Brusoni et al, 2001). Du fait de ces mutations, le modèle classique de la supply chain est profondément remis en cause.

On assiste en effet au sein de la supply chain à une externalisation croissante des activités jugées les moins stratégiques vers des sous-traitants de rang supérieur ou égal à 2 avec des contraintes fortes en matière de prix, de qualité et de prise de risque financier. Pour éviter la dispersion des ressources, les fournisseurs se doivent en retour d'apporter une réponse unifiée $^{2}$ aux exigences pourtant variées de multiples donneurs d'ordres. Simultanément, le rôle des partenaires majeurs (systémiers, équipementiers) se renforce car ils se voient confier l'ensemble des phases d'études, de développement, d'industrialisation et de réalisation d'une composante importante de l'avion. Il peut s'agir d'un module complet (fuselage, portes, nacelles, moteurs, trains d'atterrissage, systèmes de pilotage), d'équipements entrant dans la composition d'un module (équipements de cockpit, systèmes de conditionnement d'air) ou d'équipements relativement standardisés (systèmes vidéo, meubles avionique). Parmi ces partenaires majeurs émergent des firmes dites «pivots » qui ont développé des compétences particulières (Kechidi, 2008 ; Cagli et al, 2009)³. Ces compétences sont, d'une part, d'ordre

\footnotetext{
${ }^{1}$ Nous utilisons ici les résultats d'un contrat de recherche cofinancé par les Régions Aquitaine et Midi-Pyrénées et réalisé entre 2008 et 2010. Ce contrat se donnait pour objectif d'analyser l'émergence dans le tissu aéronautique régional de la forme organisationnelle que constitue la firme-pivot en réponse aux mutations qu'a connu le secteur aéronautique. A été notamment menée une analyse qualitative essentiellement fondée sur les données recueillies soit auprès d'Airbus, soit auprès de firmes-pivots, soit auprès de responsables institutionnel. Les questionnaires ont été administrés lors d'entretiens semi-directifs effectués entre mars et juin 2009. Les quarante personnes interrogées occupaient le poste de premier responsable de l'entreprise ou des fonctions directement liées à la supply chain (qualité, achats, production, stratégie,...).

${ }^{2}$ Par une modularisation accrue des productions, tout particulièrement.

${ }^{3}$ Déjà, les analyses en terme de réseau stratégique ont dans le passé développé l'idée de « hub firm », firme noyau contrôlant le réseau (Jarillo, 1988 ; Frery 1997), de broker (Miles et Snow, 1992), de firme coordinatrice manageant une chaîne de valeur (Fulconis et Paché, 2005) ou bien de firme focale organisant la spécialisation
} 
technique et portent sur un bloc de savoirs homogènes et stratégiques, et d'autre part, d'ordre relationnel et consistent en l'animation par la firme-pivot d'un réseau de sous-traitants prenant part à la conception et à la production de modules constitutifs de l'avion.

Du fait de ces mutations, la proximité organisationnelle au sein de la supply chain prend la forme d'un réseau pyramidal avec à son sommet les architectes-intégrateurs (Kechidi, Talbot, 2010). Viennent ensuite les systémiers ou intégrateur de modules, entreprises industrielles ou de prestations de services qui participent à la conception et à la réalisation d'un ensemble technique dont elles ont la responsabilité. Un systémier partage le risque financier avec l'avionneur en finançant sa R\&D et les coûts d'industrialisation et de certification des équipements. Sur le plan organisationnel, le rôle du systémier s'étend à l'approvisionnement des sous-ensembles techniques qu'il lui revient de développer, tout en veillant ensuite à leur intégration finale dans le produit avion. Cette relation globale avec le donneur d'ordres est une relation souvent ancienne fondée sur les acquis de la coopération autour des précédents programmes ( $c f$. figure 1 dans laquelle ce type de relation est schématisé en (1)). Si les systémiers détiennent des compétences techniques spécifiques mobilisables pour la conception et/ou la production d'un ensemble technique majeur, la firme-pivot s'en distingue car, en lien avec l'avionneur, elle participe à la co-spécification des produits (2). Lors de la préparation des projets, l'intervention de la firme-pivot est requise de plus en plus tôt par l'architecte-intégrateur, dans des phases très en amont et aux côtés d'autres fournisseurs majeurs potentiels en compétition. Il s'agit là de phases préparatoires, visant à la prédéfinition des projets sur la base de confrontations et d'échanges entre les acteurs industriels impliqués. Ces échanges visent à la production de connaissances communes indispensables au développement d'un sous-ensemble technique: on parle alors de co-spécification. Ces connaissances techniques sont spécifiques à un bloc de savoirs homogènes (aérostructrures, avionique, train d'atterrissage, etc.). Sont aussi produites des connaissances architecturales qui se rapportent à la manière dont les composants sont intégrés et liés entre eux dans un ensemble cohérent (Henderson, Clark, 1990). C'est à l'issue de ces processus relationnels plus ou moins coopératifs qu'une firme-pivot est sélectionnée comme fournisseur officiel de l'architecte-intégrateur.

Une fois le sous-ensemble co-spécifié avec l'architecte-intégrateur, ce dernier attend de la firme-pivot, qui restera son seul interlocuteur, qu'elle lui fournisse le sous-ensemble en 
respectant les caractéristiques techniques définies, dans les délais et au prix fixés. A charge pour la firme-pivot de faire appel, ou non, en fonction de sa stratégie d'externalisation, à des firmes de rangs équivalents ou inférieurs dans la chaîne de sous-traitance. Elle s'emploie alors à animer un réseau de sous-traitants et de firmes partenaires, en lieu et place de l'architecteintégrateur. Autrement dit, la firme-pivot doit être capable de tenir le rôle d'architecte, non pas comme l'architecte-intégrateur pour l'ensemble d'un avion, mais pour un sous-système. Elle peut faire appel à la fois à d'autres firmes-pivots et à des sous-traitants de rang inférieurs (Zuliani, 2008) :

- dans le premier cas, la relation peut épouser les mêmes principes que le lien de cospécification établi entre l'architecte-intégrateur et la firme-pivot (3). Ici fonctionne un partage des risques financiers lié à l'engagement mutuel dans un programme aéronautique. Il peut s'agir aussi d'une relation marchande classique, la firme-pivot achetant «sur étagère » les composants qui lui font défaut, sans partage de risques ni co-spécification (4);

- dans le second cas, le risk sharing ne s'applique pas dans le cadre de relations marchandes (5). On distinguera les sous-traitants de spécialité, détenteurs de savoir-faire spécifiques nourris en interne par des processus d'innovation, des sous-traitants de capacité, sélectionnés uniquement sur la base de leurs coûts de production ou de prestation.

$\mathrm{Au}$ final, les firmes-pivots apparaissent comme des architectes-intégrateurs d'un volume croissant de sous-ensembles complets, même si le degré de complétude des projets confiés à ces fournisseurs majeurs varie selon les avionneurs ${ }^{4}$. En ce sens, elles développent des proximités organisationnelles particulières basées sur une capacité à combiner des technologies, des produits et à coordonner les acteurs qui développent ces derniers.

Dans les faits, cette organisation s'avère complexe tant les relations verticales se doublent parfois de relations horizontales, une firme-pivot pouvant à la fois fournir un système complet à l'avionneur et un sous-système à un autre sous-traitant de rang 1 du même avionneur (6), tandis que certains sous-traitants de spécialité restent en lien direct avec la firme architecte (7). Une structure maillée se fait progressivement jour, pas toujours connue dans sa totalité

\footnotetext{
${ }^{4}$ En la circonstance, la délégation de systèmes complets semble singulièrement plus poussée avec Boeing ou les spécialistes d'avions régionaux Bombardier ou Embraer qu'avec Airbus (Zuliani, 2008).
} 
par l'architecte-intégrateur comme l'ont montré nos entretiens. Parfois, cette proximité organisationnelle est articulée à une proximité géographique.

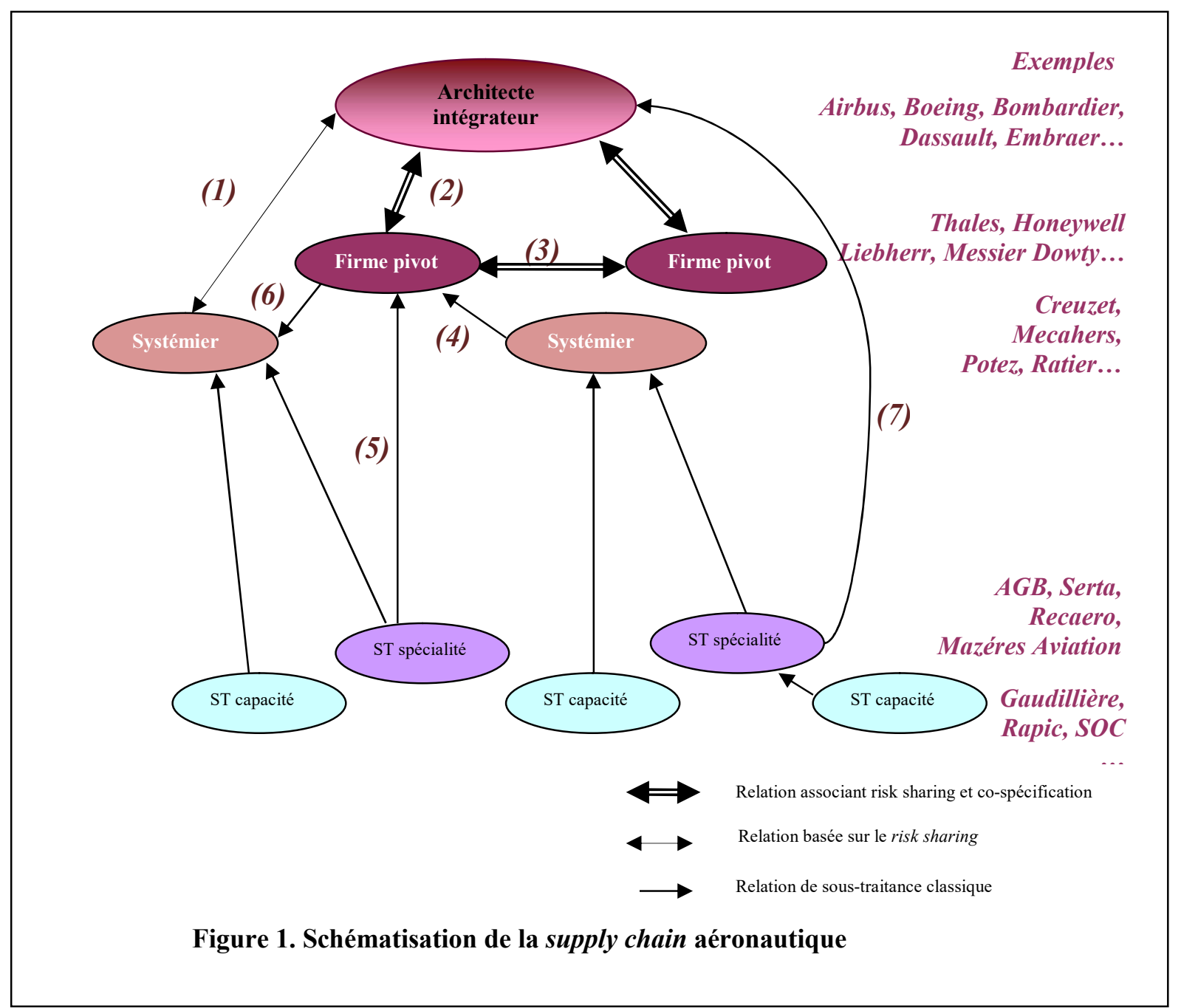

Source : auteur.

\subsection{La proximité géographique dans la supply chain}

La proximité géographique aborde la question des conditions de localisation des activités (Pecqueur et Zimmermann, 2004). Elle est un jugement qualitatif, forcément subjectif et difficilement mesurable, d'une faible distance géographique. Qualitative, elle devient une unité de mesure de l'ordre du social, cette unité prenant deux valeurs fondamentales, "être proche de », « être loin de », constituant les extrémités d'un même continuum. Lorsqu'elle est souhaitée, la proximité géographique par ses effets favorise l'émergence et/ou le renforcement d'une relation de deux façons au moins. 
En premier lieu, une proximité géographique souhaitée facilite les interactions en face à face. Nous renvoyons à l'idée classique selon laquelle l'espace physique est structuré par des infrastructures de transport et de communication. Il constitue alors un cadre matériel favorisant la circulation des informations, des biens physiques, des individus et facilitant les interactions en face à face. Dans le cas d'une proximité géographique permanente, ces dernières deviennent possibles à organiser dans un délai très bref. Le face à face peut aussi être obtenu par une proximité géographique temporaire au sein de l'entreprise (réunions, équipes plateaux) ou de temporary clusters (foires, conférences) (Carrincazeaux et Lung, 1998 ; Torre, 2009).

En second lieu, l'espace dans lequel se déploie cette proximité constitue un référent cognitif. Tous les acteurs vivent au sein de groupes situés dans un même espace géographique et associent à une aire géographique des valeurs, des représentations, des coutumes, des modes de vie, une histoire, un nom, des limites physiques et administratives, une mémoire des coordinations précédentes réussies ou échouées, des conflits, etc. L'espace joue alors un rôle dans la démarche de typification, démarche qui consiste à identifier des personnes, des objets, des actions à des «types » généralisables (Lagroye, François, Sawicki, 2006). Par exemple, Toulouse a été et est encore associée aux pères fondateurs de l'industrie aéronautique française - Morane, Dewoitine, Latécoère, - et reste la ville où sont assemblés la majorité des avions de la gamme Airbus et où sont effectués les premiers essais en vol des nouveaux programmes - Caravelle, Concorde, famille Airbus. Même si cela est moins vrai aujourd'hui, les industriels toulousains, qu'ils soient donneurs ou preneurs d'ordres, ont utilisé cette image positive dans leurs relations productives. Finalement, le lieu est à l'origine d'un sentiment d'appartenance: s'en réclamer revient à se réclamer d'un groupe social, par association (Giddens, 1987).

Sur le plan empirique, à condition d'élaborer des outils de coordination qui compensent la dispersion dans l'espace des sites, la proximité géographique n'est pas un élément indispensable à la production d'un produit complexe comme le montre la dispersion spatiale des sites européens d'Airbus. Elle n'est pas non plus indispensable à l'innovation en commun (Loilier, 2010). Si les clusters européens de l'aéronautique (Toulouse, Hambourg, Filton, Broughton, Madrid...) développent des relations d'innovation entre eux du fait de l'action structurante d'un avionneur comme Airbus (Lublinski 2003 ; Hickie, 2006), force est de constater que, paradoxalement, le développement de connaissances au sein de ces mêmes clusters est relativement moins intense (Cooke et Ehret, 2009 ; Warriner et Mullhern, 2009). 
Les firmes du secteur semblent plus s'insérer dans des réseaux a-spatiaux que territoriaux, mobilisant des savoirs et des compétences dispersés pour innover. Ce constat montre que, contrairement à l'idée selon laquelle la co-localisation conduirait systématiquement les firmes à innover de concert, il arrive souvent que les entreprises privilégient les relations à distance plutôt qu'avec ses voisins (Torre et Rallet, 2005). En effet, si la proximité géographique reste essentielle pour le transfert de connaissances, cette contrainte peut être satisfaite par la mobilité des individus et/ou des formes temporaires de proximité (équipes plateaux, réunions...) (Torre, 2008). Ainsi, cette proximité peut s'avérer utile lors des phases de conception de l'appareil car le face à face facilite grandement la recombinaison des compétences dispersées en permettant le transfert de connaissances (Boschma, 2005). En particulier, être proche géographiquement facilite l'innovation du fait d'une captation plus facile des connaissances tacites par le biais des interactions de face à face "pour révéler et combiner des savoirs tacites par essais, erreurs et tâtonnements » (Colletis et Pecqueur, 2005, p. 60). En outre, ces interactions demeurent essentielles pour répondre à des questions techniques et productives nouvelles car il permet de fluidifier les relations et de rendre les discussions plus interactives (Rallet et Torre, 2007).

Ces arguments expliquent, en partie seulement car il faut aussi y associer des raisons relatives au contrôle ( $c f$. paragraphes suivants), la constitution d'équipes plateaux en conception qui suppose une proximité géographique entre les salariés des différents sites des architectesintégrateurs et de leurs fournisseurs. L'existence d'une telle proximité géographique est illustrée par le cas toulousain ${ }^{5}$. C'est à l'occasion du développement de l'A340-500/600 en 1997 qu'Airbus a développé sa première équipe plateau. L'A380 approfondit cette organisation avec la construction d'un plateau d'un millier d'ingénieurs et de techniciens à Toulouse où sont réunis les personnels de l'avionneur, mais aussi des équipementiers. Cette période de quelques mois permet de fixer l'architecture globale de l'appareil, de co-spécifier les interfaces entre les modules et de mettre au point les solutions techniques retenues.

Parfois le besoin de face à face concerne aussi les phases d'assemblage, conduisant les principaux fournisseurs à préférer une proximité géographique permanente auprès des architectes intégrateurs. Ainsi, le développement de l'A380 au début des années 2000 a été le révélateur d'une inflexion dans le processus de localisation de certains équipementiers ${ }^{6}$ qui

\footnotetext{
5 Pour un retour sur la trajectoire du complexe aéronautique toulousain, $c f$. pour les contributions les plus récentes Hickie (2006), Leriche et Zuliani (2007) ou encore Jalabert et Zuliani (2009).

${ }^{6}$ Pour une étude approfondie des mouvements d'internationalisation des équipementiers ASD - Aéronautique, Spatial, Défense, $c f$. Carrincazeaux et Frigant (2006).
} 
s'implantent autour des sites d'Airbus pour au moins trois raisons. En premier lieu, l'assemblage renvoie à l'ensemble de la durée de vie d'un programme qui peut se compter en quelques dizaines d'année en cas de succès. En deuxième lieu les livraisons se heurtent à la taille importante des modules. En troisième lieu, la nécessaire participation aux équipes plateaux en est ainsi facilitée. C'est ainsi que s'explique la multiplication des réalisations de zones industrielles dédiées à l'aéronautique. Selon Leriche et Zuliani (2007), il existe une concentration d'équipementiers aéronautiques autour de Toulouse en raison de la présence des sites d'assemblage des Airbus A320, A330/340 et A380, de Madrid en raison de la présence d'EADS-CASA, autour des sites d'assemblage des A318/A319/A321 à Hambourg ou de production de voilures à Bristol-Filton.

Finalement, les arguments proposés ici pour expliquer la formation d'une proximité organisationnelle articulée à une proximité géographique sont d'abord d'ordre cognitif dans le sens où les acteurs sont proches pour partager des connaissances, recombiner des compétences dispersées, échanger des savoir-faire en vue d'innover ensemble. Ils doivent être complétés par l'introduction de la question du contrôle qui vient éclairer encore les raisons de la formation des proximités.

\section{CONTROLES ET PROXIMITES}

De façon générale, toute définition de la proximité, si elle peut varier selon les auteurs, donne toujours une place centrale aux interactions entre acteurs ou entre acteurs et objets, déplaçant la focale de l'individu vers l'interaction et sur la nécessité de l'existence d'un cadre cognitif commun. Les travaux proximistes font de la proximité un lien social de nature principalement cognitif, c'est-à-dire focalisant l'attention du chercheur sur les questions de traitement de l'information, de représentations, de production et de transfert de connaissances (Talbot, 2010). Les questions liées au contrôle sont encore trop ignorées. Or, il ne faut pas réduire les phénomènes de proximité à des liens sociaux apaisés et sans conflits, convergent vers une vision partagée du monde, à la poursuite d'objectifs communs acceptés et rarement remis en cause. En effet, et le constat empirique en est permanent, être à proximité c'est aussi faire face à des conflits d'intérêts, des relations de pouvoir, des remises en causes des objectifs, des tentatives de ne pas respecter ses engagements qui nécessitent un contrôle de la relation. Si l'action collective repose sur un travail cognitif de rapprochement des représentations, elle suppose pour exister un travail politique de mise en convergence des intérêts qui seront plus ou moins satisfaits par l'atteinte d'objectifs communs, d'arbitrages entre des intérêts 
inconciliables, d'imposition de choix qui apparaissent aux acteurs comme finalement légitimes, de régulation des conflits, etc. Bref, les acteurs, pour se coordonner, s'opposent autant qu'ils s'associent. La question du contrôle par la proximité se pose donc.

\subsection{Contrôles et proximité organisationnelle}

Le contrôle est une relation particulière par laquelle un individu ou une organisation influence l'action d'un autre individu ou organisation dans le sens de ses intérêts (Nogatchewsky, 2003). Il vise à dissuader les comportements opportunistes et à préserver la convergence des actions. Deux types de contrôle sont observables. Le contrôle formel prend corps dans certains critères de sélection, des règles, des dispositifs de suivi et d'évaluation, des objectifs, etc. bref des procédures qui permettent à l'acteur de s'assurer que les actions de ses partenaires sont en conformité avec ses objectifs et intérêts (Nogatchewsky, 2003 ; Brulhart, Favoreu, 2006). Ces procédures partagées et/ou imposées décrivant la marche à suivre pour assurer le contrôle, sont autant de ressources cognitives et d'outils de régulation qui rendent le contrôle effectif.

Le contrôle social est plus informel et donc moins visible. Il s'exerce à travers les réseaux sociaux d'acteurs, autre type de proximité organisationnelle. Ces réseaux sont fondés autour du partage de ressources cognitives comme des coutumes, des valeurs, de la confiance. Le partage de ces ressources conditionne d'ailleurs l'accès au réseau. Tout comportement opportuniste est sanctionné par une perte de la confiance octroyée à l'acteur par les membres du réseau, par une réputation dégradée, voire par une exclusion du réseau social. L'effet dissuasif est réel et constitue un premier aspect d'un contrôle social par la menace d'une sanction, ici la perte de confiance voire l'exclusion. De façon plus subtile, le contrôle social s'exerce via la confiance envers autrui ${ }^{7}$. Bornarel (2008), mais aussi Fenneteau et Naro (2005), montrent que la confiance, loin de se substituer au contrôle, est un moyen de contrôler les relations entre des acteurs proches socialement en freinant les comportements opportunistes, car sa perte comporte un coût social élevé. Si le contrôle consiste à influencer le comportement de quelqu'un, la confiance devient alors un mécanisme indirect de contrôle. Le recours à la confiance semble davantage se justifier dans les situations très incertaines et

\footnotetext{
${ }^{7}$ Qu'il faut distinguer selon Dupuy et Torre (2004) de la confiance en l'avenir (au sens anglo-saxon de « confidence »), celle qui fait qu'un individu accepte d'agir malgré l'incertitude et les risques inhérents à toute action.
} 
de fortes interdépendances : lorsque le contrôle hiérarchique du comportement et des résultats sont limités, la confiance devient une solution informelle, implicite, pour assurer le contrôle.

Cette confiance envers autrui est d'ordre communautaire, attachée à un individu ou un groupe social : son existence autorise les interactions au sein d'une organisation. En ce sens elle est un ingrédient essentiel aux relations sociales. Elle est générée par le partage de ressources cognitives comme des représentations, des valeurs, une mémoire des coordinations déjà réussies et par l'existence d'outils de régulation des interactions qui augmente la probabilité de réussite de ces dernières : la confiance est donc notamment générée par la proximité organisationnelle, contribuant par là-même au contrôle social.

En résumé, la proximité organisationnelle, par les procédures qu'elle fait partager rend le contrôle formel possible, par la confiance qu'elle génère contribue au contrôle social. Une fois établi, le contrôle permet d'enclencher le processus d'interactions sociales qui conduit à son tour à nourrir la confiance entre les partenaires (Philippart, 2005) dans un processus d'autorenforcement. Il n'est plus alors compris comme un frein aux interactions ; au contraire il joue un rôle stratégique dans l'instauration par exemple de relations productives entre firmes, à l'instar de ce qu'on observe dans les supply chain.

\subsection{Contrôles et proximité géographique}

De la même façon que le contrôle est renforcé par l'existence d'une proximité organisationnelle, les acteurs peuvent utiliser les effets de la proximité géographique pour l'exercer et ce de deux façons. D'une part, le face à face est l'occasion d'améliorer l'efficacité $\mathrm{du}$ contrôle formel des relations productives car il permet de rendre les discussions plus interactives et de réduire l'incertitude issue de la dimension tacite inhérente à tous savoirs et les risques d'opportunisme (Boschma, 2005). D'autre part, la proximité géographique favorise indirectement le contrôle social pour deux raisons. Premièrement, les réseaux sociaux sont souvent localisés. Comme le soulignent Bouba-Olga et Grossetti (2008), la création et le maintien des relations sociales supposent de fréquentes interactions de face à face relativement routinières qui ne peuvent pas être trop dispersées. Ceci explique qu'une part importante des réseaux sociaux se concentre dans un espace. Deuxièmement, les relations économiques sont elles-mêmes insérées ${ }^{8}$ dans ces réseaux sociaux localisés. Les membres des organisations productives, parce qu'ils appartiennent simultanément à des réseaux sociaux

\footnotetext{
${ }^{8}$ Embedded au sens de Granovetter (1985).
} 
localisés, ont alors plus à perdre à adopter une stratégie opportuniste dans leurs interactions économiques : il est plus facile de tromper un inconnu que son voisin avec lequel on partage un même réseau d'amis, d'anciens étudiants, de parents d'élèves, etc. La proximité géographique viendrait en quelque sorte verrouiller la relation entre des agents qui auraient à payer dans leur relation personnelle des comportements opportunistes adoptés dans un cadre économique. Le gain, par exemple financier, retiré d'un comportement opportuniste est dans ce cas contrebalancé par une perte plus coûteuse sur le plan social et psychologique. La mise à l'écart d'une communauté localisée transforme alors une proximité géographique souhaitée présentant des effets positifs en une proximité géographique subie source de conflits. Cette technique de contrôle social par l'appartenance à une collectivité localisée et la solidarité entre pairs est couramment pratiquée dans les districts industriels.

$\mathrm{Au}$ final, et le tableau ci-dessous synthétise notre propos, l'existence d'une proximité géographique ne doit pas être interprétée comme l'assurance qu'une relation (de contrôle par exemple) s'instaure, même si elle lui ouvre la voie. La proximité géographique ne joue que dans son articulation à une proximité organisationnelle, qu'elle soutient, renforce, compense, voire détruit en s'avérant être une source de conflits. La proximité organisationnelle quant à elle peut conduire au contrôle. Et il pourra être éventuellement renforcé par une proximité géographique.

Tableau 1 Contrôles et proximités

\begin{tabular}{|l|l|l|}
\cline { 2 - 3 } \multicolumn{1}{c|}{} & \multicolumn{1}{c|}{ Contrôle formel } & \multicolumn{1}{c|}{ Contrôle social } \\
\hline $\begin{array}{l}\text { Proximité } \\
\text { organisationnelle }\end{array}$ & $\begin{array}{l}\text { Conduit au contrôle via le partage de } \\
\text { procédures }\end{array}$ & Conduit au contrôle via la confiance \\
\hline $\begin{array}{l}\text { Proximité } \\
\text { géographique }\end{array}$ & $\begin{array}{l}\text { Renforce ce contrôle en facilitant les } \\
\text { face à face }\end{array}$ & $\begin{array}{l}\text { Renforce ce contrôle car les réseaux } \\
\text { sociaux, dans lesquels sont encastrées } \\
\text { les relations économiques, sont } \\
\text { souvent localisés }\end{array}$ \\
\hline
\end{tabular}

Source : auteur. 


\subsection{Contrôles et proximités dans la chaine d'approvisionnement d'Airbus}

Certains acteurs majeurs du secteur aéronautique mobilisent la proximité organisationnelle qui se déploie au sein de la supply chain pour exercer un contrôle. Les firmes architectes parviennent ainsi à maintenir un degré élevé de contrôle des participants à différentes stades de la chaîne de valeur autrement que par intégration patrimoniale (Busby, Fan, 1993 ; Frery, 1997), tout en permettant le co-développement en général (Garel, 1999) et la co-conception en particulier (Takeishi, 2001 ; O'Sullivan, 2006).

Ce contrôle, par nature difficilement observable, prend corps au sein de divers outils de régulation de la relation client fournisseur. Dans une supply chain, les industriels ont à la fois des intérêts convergents car ils doivent «faire ensemble» le produit final, mais aussi divergents car ils cherchent à capter la plus grande part de la valeur ajoutée (Nogatchewsky, 2006). De ce fait, il faut développer des dispositifs permettant de se prémunir de l'opportunisme de chacun, de s'assurer des compétences des fournisseurs et de réduire l'asymétrie d'information. Concrètement sont examinés l'aptitude à la qualité, la capacité d'innovation, la pérennité financière, etc. Des mécanismes de suivi de la performance des fournisseurs (qualité, coûts, délais) et d'amélioration des process complètent le dispositif. Les dispositifs d'incitation de réduction des coûts planifient dans le temps la réduction des coûts d'achats des donneurs d'ordres. Les dispositifs de protection mis en place par le client via les contrats d'exclusivité, les accords de confidentialité, les audits de surveillance, visent à se protéger de l'opportunisme du fournisseur et à assurer la continuité des échanges. De même, l'immixtion du fournisseur chez le client est elle aussi maitrisée, par exemple en réglementant la circulation au sein des plateaux et les visites ponctuelles en vue de canaliser les informations livrées.

De tels outils de contrôle ont été mis en place au sein de la supply chain aéronautique européenne. Pour opérationnaliser la stratégie d'externalisation développée par les architectes-intégrateurs, un important effort de coordination s'avère nécessaire tout particulièrement dans le domaine de l'échange d'informations (Terwiesch, Loch, De Meyer, 2002). De nombreux outils de coordination à distance sont alors élaborés notamment grâce aux TIC: maquettes numériques créant des plateaux virtuels, EDI, etc. Mais ces outils peuvent s'avérer insuffisants pour permettre le transfert de connaissances, la recombinaison des compétences et le contrôle la supply chain. Ils doivent alors être complétés par des outils régulant les rencontres, associant proximité organisationnelle et proximité géographique temporaire. 
Cagli et al, 2009 constatent qu'une perspective d'affaires entre Airbus et une firme-pivot met en relation deux équipes constituées chez chacun des partenaires et qui organisent de fréquents face à face. Ces équipes sont un élément essentiel de la réalisation du contrôle. Selon les auteurs, elles se réunissent dans un cadre organisationnel très précis qui s'articule en plusieurs temps.

Un premier temps, long (5 à 6 ans), très en amont de la fabrication d'un avion, renvoie à des collaborations plus ou moins intenses, plus ou moins organisées. Les besoins du marché et les techniques disponibles pour y répondre sont examinés. A ce stade, aucune contractualisation n'est encore envisagée, seule la confidentialité étant exigée. Le plus souvent, le dialogue est organisé à distance, la proximité géographique jouant un faible rôle ici. Du point de vue du contrôle, les partenaires évaluent les capacités de chacun et cherchent à construire une relation de confiance.

Du côté des fournisseurs, le systémier est associé très tôt aux phases conceptrices dites de cospécification. Par exemple, Thales Avionics co-définit avec Airbus les systèmes possibles, leurs objectifs techniques et de coûts. L'objectif de Thales Avionics est d'échapper autant que faire se peut au contrôle d'Airbus par la maîtrise la plus complète possible, tant de la conception que de la production de systèmes avioniques qu'elle a en charge. L'enjeu pour ce fournisseur de rang 1 est bien de peser plus ou moins intensément dans le sens de ses intérêts sur les choix techniques réalisés. La relation se veut alors moins asymétrique et vient soutenir la stratégie de Thales de remontée dans la chaîne de valeur. Du côté des architectesintégrateurs, il s'agit de jauger de la crédibilité des futurs engagements qui seront pris tout en captant des connaissances utiles au développement du programme. Le contrôle est donc très informel durant cette phase d'avant-plateau : il est plus social que légal, un comportement opportuniste du fournisseur pouvant le conduire à ne pas être retenu par Airbus.

Dans un deuxième temps en effet, ajoutent les auteurs, l'avionneur européen soit retient une «short list» soit sélectionne un seul fournisseur par module. Chaque participant au programme doit démontrer la crédibilité des systèmes qu'il fournit. Concrètement, une proposition commerciale et des plannings de réalisation sont posés. Le contrôle prend un aspect plus formel, se présentant sous la forme de critères de sélection, d'une conformité aux spécifications techniques, d'engagements dans le temps et de respect des prix. Sont mises en perspective les capacités techniques, financières et organisationnelles des preneurs d'ordres avec leurs engagements : le contrôle porte à la fois sur la mise en conformité avec les 
spécifications demandées et la crédibilité de la réponse fournie. Il devient ici très formel car objectivable et génère de la confiance. Il permet à la réalisation productive de débuter.

Dans un troisième temps, et une fois la proposition acceptée par Airbus, débute la phase très technique des équipes plateaux durant laquelle les prototypes de modules sont réalisés. Ici la proximité géographique vient renforcer le contrôle d'une relation fondée sur un face à face permanent puisque les équipes sont co-localisées pour plusieurs mois (de 4 à 6 mois en général). La relation productive est ici contrôlée par des dispositifs techniques, commerciaux et légaux. Cela signifie que des critères de performance en termes de prix, qualité et délais sont construits et figés pour faciliter le contrôle lors de l'industrialisation future du programme. Là encore, puisque la relation se formalise, le contrôle formel prédomine.

Parfois la proximité organisationnelle peut s'articuler à une proximité géographique permanente, le fournisseur de rang 1 choisissant de se localiser à demeure près des sites d'assemblage d'Airbus, comme ceux de Toulouse. L'affirmation de Toulouse comme capitale industrielle de l'avionneur européen justifie le choix stratégique des équipementiers, en particulier pour les industriels qui s'engagent pour la première fois de façon majeure auprès d'Airbus. Lors du lancement du programme A380, c'était notamment le cas pour les équipementiers américains traditionnellement plus attachés au concurrent Boeing. Rockwell Collins pour certains systèmes d'avionique (radio navigation), Goodrich pour les nacelles de réacteurs et les inverseurs de poussée, se sont installés ou ont renforcé leur présence à Toulouse dès le début du programme, non seulement pour profiter des possibilités de fréquentes interactions en face à face, mais aussi car la réalisation d'investissements à proximité du centre de décision d'Airbus constitue une preuve d'engagement dans la relation en offrant des opportunités accrues de contrôle.

\section{CONCLUSION}

Finalement, la proximité est un vecteur du contrôle du partenaire. Il est en effet apparu que le contrôle, qu'il soit formel ou informel, devenait possible s'il s'accompagnait d'une proximité organisationnelle entre le contrôleur et le contrôlé. Nous avons aussi analysé en quoi la proximité géographique peut venir renforcer ce contrôle. Pour illustrer ce propos théorique sur le contrôle par les proximités, divers outils de coordination utilisés dans la supply chain aéronautique ont été analysés. Nous avons montré que ces outils, explicitement présentés 
comme favorisant le partage de connaissances et la recombinaison des compétences, permettent implicitement tout autant le contrôle. Mais il reste au final très difficile de distinguer autrement qu'analytiquement ce qui relève d'une volonté de diffuser des connaissances de ce qui relève d'une stratégie visant à rendre le contrôle effectif, tant les outils pour rendre effectifs ce partage et ce contrôle sont souvent les mêmes. Pourtant, les deux phénomènes doivent être discernés, ce qui peut passer par la construction d'indicateurs. Au-delà de ce travail théorique d'approfondissement, un travail cette fois de généralisation de nos résultats à d'autres secteurs doit être entrepris. L'industrie automobile semble prometteuse pour une première généralisation, tant elle présente au moins au niveau de la supply chain des similitudes avec l'industrie aéronautique comme nous l'avons montré par ailleurs (Frigant, Talbot, 2005). D'autant qu'il existe déjà des travaux concernant le contrôle de la supply chain dans les activités automobiles (cf. par exemple, Philippart, 2005 ; Pernot et Roodhooft, 2008). 


\section{BIBLIOGRAPHIE}

Bornarel, F., (2008), Relations de confiance et renforcement du contrôle, Finance Contrôle Stratégie, vol. $11 \mathrm{n}^{\circ} 1$, pp. 71-104.

Boschma, R., (2005), Does geographical proximity favour innovation?, Economie et Institutions, $\mathrm{n}^{\circ} 6$ et 7 , pp. 111-128.

Bouba-Olga, O., Grossetti, M., (2008), Socio-économie de proximité, Revue d'Economie Régionale et Urbaine, ${ }^{\circ} 3$, pp. 311-328.

Brulhart, F., Favoreu, C., (2006), Le lien contrôle-confiance-performance dans les relations de partenariat logistique inter-firmes, Finance Contrôle Stratégie, vol. 9 n5, pp. 59-96.

Brusoni, S., Prencipe A., (2001), Unpacking the Black Box of Modularity: Technologies, Products and Organizations, Industrial and Corporate Change, vol. $10 \mathrm{n}$ 1, pp. 179-204.

Brusoni, S., Prencipe, A., Pavitt, K., (2001), Knowledge Specialisation, Organizational Coupling, and the Boundaries of the Firm: Why Do Firms Know More Than They Make?, Administrative Science Quarterly, vol. 46 n4, pp. 597-621.

Busby, J.S., Fan, I.S. (1993), The Extended Manufacturing Enterprise: Its Nature and its Need, International Journal of Technology Management, vol. $8 \mathrm{n}^{\circ}$ 3/4/5, pp. 294-308.

Cagli, A., Kechidi, M., Lévy, R., (2009), Gestion stratégique de la supply chain et firme-pivot dans le secteur aéronautique, Revue Française de Gestion Industrielle, ${ }^{\circ} 2$, juin.

Colletis, G., Pecqueur, B., (2005), Révélation de ressources spécifiques et coordination située, Economie et Institutions, ${ }^{\circ} 6$ et 7, 1er et 2e semestres, pp. 51-73.

Carrincazeaux, C., Frigant, V., (2006), L'internationalisation de l'industrie aéronautiquespatiale-défense française: vers une banalisation des formes d'internationalisation?, in SESSI, L'industrie en France et la mondialisation, SESSI, Paris.

Carrincazeaux, C., Lung, Y., (1998), La proximité dans l'organisation des activités de conception des produits automobiles, in Bellet M., Kirat T. et Largeron C. (Eds.), Approches multiformes de la proximité, Hermès, Paris.

Cooke, P., Ehret, O., (2009), Proximity and Procurement: A Study of Agglomeration in the Welsh Aerospace Industry, European Planning Studies, vol. 17 n4, pp. 549-567.

Das, T.K., Teng, B.S., (2001), Trust, Control and Risk in Strategic Alliances: An Integrated Framework, Organization Studies, vol. 22 n², pp. 251-283.

Dekker, H., (2004), Control of inter-organisational relationships: evidence on appropriation concerns and coordination requirements, Accounting, Organizations and Society, vol. 29, pp. 27-49.

Dupuy, C., Torre, A., (2004), Confiance et Proximité, in Pecqueur B. et Zimmermann J.B. (Eds), Economie de Proximités, Hermès, Lavoisier, Paris.

Fenneteau, H., Naro, G., (2005), Contrôle et confiance dans l'entreprise virtuelle. Illustrations logistiques, Revue française de gestion, vol. 3 n 156, pp. 203-219.

Frenken, K., (2000), A complexity approach to innovation networks. The case of the aircraft industry (1909-1997), Research Policy, vol. 29 n², pp. 257-272.

Frery, F., (1997), Le contrôle des réseaux d'entreprises : pour une extension du concept d'entreprise, Actes de la Conférence AIMS, Montréal, juin.

Frigant, V., Talbot, D., (2005), "Technological Determinism and Modularity: Lessons from a Comparison between Aircraft and Auto Industries in Europe", Industry and Innovation, vol. $12 \mathrm{n}^{\circ} 3$, September, pp. 337-335.

Fulconis, F., Paché, G., (2005), Piloter des entreprises virtuelles : un rôle nouveau pour les prestataires de services logistiques, Revue française de gestion, vol. 3 n¹56, pp. 167-186.

Garel, G., (1999), Analyse d'une performance de co-développement, Revue Française de Gestion, mars-avril-mai, pp. 63-91. 
Giddens, A., (1987), La constitution de la société, Presses Universitaires de France, Paris.

Granovetter, M., (1985), Economic action and social structure: the problem of embeddedness, The American Journal of Sociology, vol. 91 n³, pp. 481-510.

Guilhon, B., Gianfaldoni, P., (1990), Chaînes de compétences et réseaux, Revue d'Economie Industrielle, $\mathrm{n}^{\circ} 51, \mathrm{pp}$. 97-112.

Henderson, R., Clark, K., (1990), Architectural innovation: the reconfiguration of existing product technologies and the failure of established firms, Administrative Science Quaterly, vol. $35 \mathrm{n}^{\circ} 1$, pp. 9-30.

Hickie, D., (2006), Knowledge and competitiveness in the aerospace industry: the cases of Toulouse, Seattle and North-west England, European Planning Studies, vol. 14 n5, pp. 697716.

Jalabert, G., Zuliani, J.M., (2009), Toulouse, l'avion et la ville, Privat, Toulouse.

Jarillo, J.C., (1988), On strategic networks, Strategic Management Journal, vol. 9, pp. 31-41.

Kechidi, M., (2008), Modularité, firme-pivot et innovations: le cas d'Airbus, Revue Française de Gestion Industrielle, vol. 27, pp. 21-40.

Kechidi, M., Talbot, D., (2010), Institutions and coordination: what is the contribution of a proximity-based analysis? The case of Airbus and its relations with the subcontracting network, International Journal of Technology Management, vol. $50 \mathrm{n}^{\circ} 3 / 4$, pp. 285-299.

Lagroye, J., François, B., Sawicki, F., (2006), Sociologie Politique, $5^{\mathrm{e}}$ édition, Presses de Sciences Po et Dalloz, Paris.

Lauriol, J., Perret, V., Tannery, F., (2008), Stratégies, espaces et territoires. Une introduction sous le prisme géographique, Revue Française de Gestion, vol. 4 n¹84, pp. 91-103.

Leriche, F., Zuliani, J.M., (2007), L'industrie aéronautique toulousaine et la déplétion pétrolière : quelles perspectives ?, Géographie, Economie, Société, n 9 , pp. 19-38.

Loilier, T., (2010), Innovation et territoire. Le rôle de la proximité géographique ne doit pas être surestimé, Revue Française de Gestion, vol. 4 n²00, pp. 15-35.

Lublinski, A.E., (2003), Does Geographic Proximity Matter?. Evidence from Clustered and Non-clustered Aeronautic Firms in Germany, Regional Studies, vol. 37 n 5, pp. 453-467.

Mazaud, F., (2006), De la firme sous-traitante de premier rang à la firme-pivot. Une mutation de l'organisation du système productif, Revue d'économie industrielle, $\mathrm{n}^{\circ} 113,1^{\mathrm{er}}$ trimestre, pp. 45-60.

Miles, R., Snow, C., (1992), Causes of failure in network organizations, Californian Management Review, vol. $34 \mathrm{n}^{\circ} 4$. pp. 53-72.

Nogatchewsky, G., (2003), L'exercice du contrôle dans la relation client-fournisseur, Revue française de gestion, vol. $6 \mathrm{n}^{\circ}$ 147, pp. 173-183.

Nogatchewsky, G., (2006), L'impact de la dépendance sur les stratégies de contrôle d'un équipementier automobile vis-à-vis de ses fournisseurs: une lecture militaire, Finance Contrôle Stratégie, vol. 9 n², pp. 89-120.

Ouchi, W., (1979), A Conceptual Framework for the Design of Organizational Control Mechanisms, Management Science, vol. 25 nº $^{\circ}$, pp. 833-848.

O'Sullivan, A., (2006), Why tense, unstable, and diverse relations are inherent in codesigning with suppliers: an aerospace case study, Industrial and Corporate Change, vol. 15 n², pp. 221-250.

Pecqueur, B., Zimmermann, J.B., (2004), (Eds.), Economie de Proximités, Hermès, Lavoisier, Paris.

Pernot, E., Roodhooft, F., (2008), Management control of supplier relationships in manufacturing: A case study in the automotive industry, Vlerick Leuven Gent Management School Working Paper Series 2008-23, Vlerick Leuven Gent Management School. 
Philippart, P., (2005), La dialogique contrat-confiance dans la gestion des alliances interentreprises : une illustration dans l'industrie automobile, Finance Contrôle Stratégie, vol. $8 \mathrm{n}^{\circ} 4$, pp. 177-203.

Rallet, A., (1999), L'économie de proximités, communication à l'école-chercheur INRA, Le Croisic, 8-10 décembre.

Rallet, A., Torre, A., (2007), Introduction. Faut-il être proche pour innover ensemble ?, in Rallet, A., Torre, A., (Eds.), Quelles proximités pour innover?, L'Harmattan, Paris.

Takeishi, A., (2001), Bridging inter- and intra-firm boundaries: management of supplier involvement in automobile product development, Strategic Management Journal, vol. 22, pp. 403-433.

Talbot, D., (2010), La dimension politique dans l'approche de la proximité, Géographie, Economie, Société, vol. 22 n², pp. 125-144.

Terwiesch C., Loch C., De Meyer A. (2002), Exchanging preliminary information in concurrent engineering: alternative coordination strategies, Organization Science, vol. $13 \mathrm{n}^{\circ} 4$, pp. 402-419.

Torre, A., (2008), On the Role Played by Temporary Geographical Proximity in Knowledge Transmission, Regional Studies, vol. 42 n6, pp. 869-889.

Torre, A., (2009), Retour sur la notion de proximité géographique, Géographie, Economie, Société, vol. 11, pp. 63-75.

Torre A., Rallet A., (2005), Proximity and localization, Regional Studies, vol. 39 n¹, pp. $47-$ 60.

Van der Meer-Kooistra, J., Vosselman, E.G., (2001), Management control of interfirm transactional relationships: the case of industrial renovation and maintenance, Accounting, Organizations and Society, vol. 25, pp. 51-77.

Warriner, P., Mulhern, C., (2009), From metal bashing to materials science and services: Advanced manufacturing and mining clusters in transition, European Planning Studies, vol. $17 \mathrm{n}^{\circ} 1$, pp. $120-134$.

Zuliani, J.M., (2008), The Toulouse Cluster of On-board Systems: A Process of Collective Innovation and Learning, European Planning Studies, vol. 16 n5, pp. 711-726. 